Based on the CORONERVE Programme and latest retrospective Lancet cohort studies, the period between 14 and 90 days after diagnosis, 5.8\% COVID-19 survivors had their first recorded diagnosis of psychiatric illness.

It is also important to consider other organic disease given the simultaneous diagnosis of COVID-19. Although it is not yet possible to confirm here due to the lack of a validated CSF-PCR assay, previous reports have implicated SARS-CoV-2 in the development of viral encephalitis, and this remains an important differential.

Conclusion. Clinicians should be alert to the possibility of patients with COVID-19 developing neuropsychiatric complications post SARS-CoV-2 infection, mandating the need for vigilant initial neuropsychiatric assessment and possibly follow-up care in 3 months.

\section{The effectiveness of telepsychiatry: a thematic review}

Gunjan Sharma* and Karrish Devan

South London and Maudsley NHS Foundation Trust

${ }^{\star}$ Corresponding author.

doi: $10.1192 /$ bjo.2021.183

Aims. The authors conducted a thematic review on the effectiveness of Telepsychiatry in light of the COVID-19 pandemic. The study aimed to clarify the effectiveness of Telepsychiatry, providing an evidence base for the growing use of Telepsychiatry.

Method. The authors searched three databases - Cochrane, PubMed and PsychINFO - using the terms virtual consultation/ telepsychiatry/video consultation AND psychiatry/mental illness.

The authors excluded all papers that were not in English and that did not focus on the psychiatric consultation.

Result. 961 papers were identified, reduced to 321 using exclusion criteria and removal of duplicates. Using thematic analysis the authors found five themes that occurred across all papers in relation to the effectiveness of Telepsychiatry.

Patient \& Clinician Satisfaction

There is consistently high patient satisfaction with telepsychiatry but lower clinician satisfaction, often as a result of cynicism and a lack of familiarity. Clinician satisfaction increases when clinicians trial Telepsychiatry and become more positive about its uses.

\section{Diagnostic Reliability}

Telepsychiatry was found to have high levels of inter-rater reliability equivalent to face-to-face consultations for common disorders including mood and psychotic disorders, substance misuse and dementia. It was also found to have high levels of diagnostic reliability across age groups.

Outconfesepsychiatry has been found to reduce symptoms of common psychiatric disorders and improve quality of life in a variety of environments including emergency departments, inpatient units and prisons. Telepsychiatry increases access to specialised services resulting in quicker access to treatment and reduction in admissions.

\section{Technology}

Without adequate internet connectivity clinicians are unable to conduct an appropriate mental state examination and the therapeutic relationship becomes challenging. Inadequate technology can impact the effectiveness of Telepsychiatry amongst those who are socioeconomically disadvantaged and may not have access to appropriate technology.

\section{Professional Guidance}

There is a concerning lack of guidance around the use of Telepsychiatry. Without clear protocols there is a lack of standardisation and clinicians are unwilling to integrate Telepsychiatry into their practice. Main concerns raised are around confidentiality, consent, the appropriateness of certain patient groups and emergencies. Conclusion. This review found evidence for the effectiveness of Telepsychiatry with greatest emphasis on technology and patient satisfaction. The main barrier is the reluctance amongst clinicians to facilitate Telepsychiatry into their practice, often due to cynicism and a lack of familiarity. The authors recommend training in the uses of Telepsychiatry and the provision of professional guidance from medical bodies to allay concerns and provide clear standards.

\section{Working in the woodlands: a mixed methods evaluation of Green Care in first episode psychosis \\ Harriet Sharp ${ }^{1 \star}$, Sharon Cuthbert ${ }^{2}$ and Clio Berry ${ }^{3}$ \\ ${ }^{1}$ Sussex Partnership Nhs Foundation Trust, Brighton and Sussex Medical School; ${ }^{2}$ Sussex Partnership Nhs Foundation Trust and \\ ${ }^{3}$ Brighton and Sussex Medical School University of Sussex \\ ${ }^{\star}$ Corresponding author.}

doi: 10.1192/bjo.2021.184

Aims. Recognition of the essential role of nature-based activities for general wellbeing is expanding. Previous evaluation of naturebased activities has shown that those with greater mental health needs may benefit proportionally more compared to the general population. Currently, there is limited evidence of the benefits of green care for those with severe and enduring mental illness, including psychosis.

We aim to establish benefits and difficulties encountered during a 10-session green care programme for 18-30 year olds who have experienced first episode of psychosis (FEP) using a mixed methods approach.

Method. This was a service evaluation of the Woodland Group, run by Circle of Life Rediscovery (CLR) and commissioned by Sussex Partnership NHS Foundation Trust in Autumn 2019 for 10 half-day sessions. All participants were aged 18-30 years, referred from Early Intervention in Psychosis service and had experienced FEP. Patients were supported by EIS staff with a ratio of at least 3:1. Sessions consisted of a welcome and agenda setting, ice-breaking activity, core nature-based activity (such as roasting chestnuts, maintaining the woodland area) and a 'sense meditation'.

Quantitative data for this evaluation were collected through routinely collected 15-item Questionnaire on the Process of Recovery (QPR), and a semi-structured intervention experience questionnaire. Qualitative data were collected via a focus group within the final session of the Woodlands Group. Thematic analysis was performed by the three co-authors.

Result. Session attendance ranged between 3-15. 4/8 patients showed reliable improvement on QPR outcome measures, 1 showed deterioration and 3 showed no change. Mean QPR scores showed modest increase from average 3.4 (week 1) to 3.8 (week 10). 100\% of respondents would recommend this group to others. Thematic analysis identified themes of connection with nature and others, development of a sense of wellbeing and 'peacefulness' and new perspectives on psychotic experience.

Conclusion. This small, retrospective evaluation is the first to investigate green care interventions for young people experiencing FEP. Our results reflect the positive informal feedback from participants and supporting staff following attendance at the Woodlands Group. Limitations include small sample size, incomplete data, and reliance on patient-reported outcomes. These findings show promise for green care activities within EIS and represents a sustainable intervention in mental health care. 